\title{
THE SINGULARITIES OF THE $\mathscr{S}$-MATRIX AND GREEN'S FUNCTION ASSOCIATED WITH PERTURBATIONS OF $-\Delta$ ACTING IN A CYLINDER
}

\author{
BY CHARLES I. GOLDSTEIN ${ }^{1}$ \\ Communicated by Murray Protter, April 3, 1973
}

It is the purpose of this note to study the singularities of the $\mathscr{S}$-matrix and Green's function associated with the operators considered in [1]-[3]. As will be seen, there are a countable number of branch points, as well as a countable number of different $\mathscr{S}$-matrices associated with these operators. In this respect, these results differ considerably from those drawn from quantum mechanical scattering ${ }^{2}$ and the exterior problem (see e.g. [4] and [5]).

1. Preliminaries. Let $S$ denote the semi-infinite cylinder in $R^{N}$, $N$-dimensional Euclidean space $(N \geqq 2)$, with arbitrary bounded, smooth $N-1$ dimensional cross-section $l$. Thus $S$ consists of the points $x=$ $\left(\left(x_{1}, \ldots, x_{N-1}\right), x_{N}\right)=\left(\tilde{x}, x_{N}\right)$, where $\tilde{x} \in l$ and $x_{N} \geqq 0 .^{3}$ Let $\Omega$ denote the domain with smooth boundary $\dot{\Omega}$, obtained from $S$ by perturbing a finite part of $\dot{S}$. Thus $\Omega=S$ for $x_{N} \geqq \stackrel{\circ}{x}_{N}$ for some fixed $\stackrel{\circ}{x}_{N}>0$.

We now define the operators $A_{0}(A)$ by $-\Delta$ acting in $L_{2}(S)\left(L_{2}(\Omega)\right)$ and associated with zero Dirichlet boundary conditions on $\dot{S}(\dot{\Omega})$. Let $A_{l}$ denote the corresponding operator defined in $L_{2}(l)$ and let $\left\{v_{n}\right\}$ and $\eta_{n}(\tilde{x})$ denote a complete set of eigenvalues (in increasing order) and corresponding orthonormal eigenfunctions for $A_{l}$. Let $A^{c}$ denote that part of $A$ orthogonal to all of its eigenvalues, $\Lambda$ denote the set of eigenvalues of $A$ and $\Lambda^{\prime}=\Lambda \cup\left\{v_{n}\right\}$.

It was shown in [1] that a complete set of generalized eigenfunctions for $A_{0}$ and $A^{c}$ are given by

$$
w_{n}^{0}(x ; \lambda)=(2 / \pi)^{1 / 2} \sin \left(\lambda-v_{n}\right)^{1 / 2} x_{N} \eta_{n}(\tilde{x}), \quad \lambda \notin\left\{v_{n}\right\}
$$

AMS (MOS) subject classifications (1970). Primary 35J05, 35J10, 47A40, 78A50.

Key words and phrases. Singularities of the $\mathscr{S}$-matrix, Green's function, waveguides, infinitely sheeted Riemann surface, analytic and meromorphic continuation, radiation condition, resonant state.

${ }^{1}$ Work performed under the joint auspices of the Atomic Energy Commission and the Mathematics Research Center at the University of Wisconsin.

${ }^{2}$ Our results are related to wave progagation in a waveguide.

${ }^{3}$ We might just as easily consider the infinite cylinder, $S^{\prime}=\left(x=\left(\tilde{x}, x_{N}\right) \mid \tilde{x} \in l,-\infty<\right.$ $\left.x_{N}<\infty\right)$. 
and

where

$$
w_{n}^{-}(x ; \lambda)=w_{n}^{0}(x ; \lambda)+v_{n}^{-}(x ; \lambda), \quad \lambda \notin \Lambda^{\prime},
$$

$$
\begin{aligned}
v_{n}^{-}(x ; \lambda)= & \sum_{n^{\prime}=1}^{m} c_{n^{\prime}}^{n}(\lambda) \exp \left\{-i\left(\lambda-v_{n^{\prime}}\right)^{1 / 2} x_{N}\right\} \eta_{n^{\prime}}(\tilde{x}) \\
& +\sum_{n^{\prime}=m+1}^{\infty} c_{n^{\prime}}^{n^{-}}(\lambda) \exp \left\{-\left(v_{n^{\prime}}-\lambda\right)^{1 / 2} x_{N}\right\} \eta_{n^{\prime}}(\tilde{x})
\end{aligned}
$$

for $x_{N} \geqq \stackrel{\circ}{x}_{N}$ and $\lambda \in\left(v_{m}, v_{m+1}\right),()^{1 / 2}$ denoting the positive square root. Another complete set of generalized eigenfunctions, $w_{n}^{+}(x ; \lambda)=w_{n}^{0}(x ; \lambda)+$ $v_{n}^{+}(x ; \lambda)$, for $A^{c}$ are defined analogously with $\exp \left\{-i()^{1 / 2}\right\}$ replaced by $\exp \left\{i()^{1 / 2}\right\}$ and $c_{n^{\prime}}^{n^{-}}(\lambda)$ by $c_{n^{\prime}}^{n^{+}}(\lambda)$.

It was proven in [3] that the $\mathscr{S}$-matrix, $\mathscr{S}_{m}(\lambda)$, associated with $A_{0}$ and $A$ at the point $\lambda \in\left(v_{m}, v_{m+1}\right), \lambda \notin \Lambda$, is given by the matrix

$$
\mathscr{S}_{m}(\lambda)=I_{m}+T_{m}(\lambda),
$$

where $I_{m}$ is the identity matrix of rank $m$ and

$$
\begin{aligned}
T_{m}(\lambda) & =\left(t_{n, n^{\prime}}(\lambda)\right) \quad \text { with } \\
t_{n, n^{\prime}}(\lambda) & =-(2 \pi)^{1 / 2} i c_{n^{\prime}}^{n^{-}}(\lambda), \quad n, n^{\prime}=1, \ldots, m .
\end{aligned}
$$

Note that the rank $m$ of $\mathscr{S}_{m}(\lambda)$ varies with $\lambda$.

It follows from the arguments of [6] that Green's function, $G_{0}^{-}(x, y ; \lambda)$, for the operator $A_{0}-\lambda$ is given by

$$
G_{0}^{-}(x, y ; \lambda)=\sum_{n \neq 1}^{\infty} \frac{\sin \left(\lambda-v_{n}\right)^{1 / 2} x_{N} \exp \left\{-i\left(\lambda-v_{n}\right)^{1 / 2} y_{N}\right\}}{\left(\lambda-v_{n}\right)^{1 / 2}} \eta_{n}(\tilde{x}) \eta_{n}(\tilde{y}),
$$

$$
\begin{array}{rr}
\text { for } x_{N}<y_{N} \\
\sum_{n=1}^{\infty} \frac{\exp \left\{-i\left(\lambda-v_{n}\right)^{1 / 2} x_{N}\right\}}{\left(\lambda-v_{n}\right)^{1 / 2}} \sin \left(\lambda-v_{n}\right)^{1 / 2} y_{N} \eta_{n}(\tilde{x}) \eta_{n}(\tilde{y}) \\
\text { for } x_{N}>y_{N},
\end{array}
$$

where $x, y \in S$ and $\operatorname{Im}\left(\lambda-v_{n}\right)^{1 / 2}<0, n=1,2, \ldots$ In view of (1.1) and (1.4), it is natural to define the infinitely sheeted Riemann surface $R_{\infty}$, obtained by making each point $v_{n}$ a branch point of order one. By $\Gamma_{n_{1}, \ldots, n_{k}}\left(\mathrm{cl}\left(\Gamma_{n_{1}, \ldots, n_{k}}\right)\right)$, we shall mean that sheet of $R_{\infty}$ consisting of those points $\lambda$ for which $0<\arg \left(\lambda-v_{n}\right)<2 \pi\left(0 \leqq \arg \left(\lambda-v_{n}\right)<2 \pi\right)$ for $n=n_{1}, \ldots, n_{k}$ and $-2 \pi<\arg \left(\lambda-v_{n}\right)<0\left(-2 \pi \leqq \arg \left(\lambda-v_{n}\right)<0\right)$ for all remaining $n$. The "physical sheet", $\Gamma_{0}\left(\operatorname{cl}\left(\Gamma_{0}\right)\right)$, shall consist of those $\lambda$ satisfying $-2 \pi<\arg \left(\lambda-v_{n}\right)<0\left(-2 \pi \leqq \arg \left(\lambda-v_{n}\right)<0\right), n=1,2, \ldots$

It can be easily seen that $G_{0}^{-}(x, y ; \lambda)$, defined initially on $\Gamma_{0}$ has an analytic continuation onto all of $R_{\infty}$ in the following sense. Consider 
$\Gamma_{m}, m \geqq 1$, suppose $\mathscr{G}=[a, b] \subset\left(v_{J}, v_{J+1}\right)$ for an arbitrary $J \geqq m$ and set $\kappa=\left(\lambda-v_{m}\right)^{1 / 2}$ for each $\lambda \in \mathscr{G}$. Then the function $\tilde{G}_{0}^{-}(x, y ; \kappa) \equiv_{\mathrm{df}}$ $G_{0}^{-}(x, y ; \lambda)$ is an analytic function of $\kappa$ for each $\kappa \in \operatorname{Im} \kappa<0 \cup$ $\left[\left(a-v_{m}\right)^{1 / 2},\left(b-v_{m}\right)^{1 / 2}\right] \cup \operatorname{Im} \kappa>0$ such that $\operatorname{Im}\left(\kappa^{2}+v_{m}-v_{n}\right)^{1 / 2} \leqq$ 0 for $n \neq m$. Hence $G_{0}^{-}(x, y ; \lambda)$ has an analytic continuation from $\Gamma_{0}$ onto $\Gamma_{m}$ across $\mathscr{G}$. We define an analytic (meromorphic) continuation of a complex or operator-valued function $F(\lambda)$ from an arbitrary sheet of $R_{\infty}$ onto any other sheet in an analogous fashion. If such an analytic (meromorphic) continuation exists for each sheet of $R_{\infty}$, we say that $F(\lambda)$ is analytic (meromorphic) on $R_{\infty}$. We shall say that $\lambda_{0} \in R_{\infty}$ is a pole of $F(\lambda)$ if $\kappa_{0}=\left(\lambda_{0}-v_{m}\right)^{1 / 2}$ is a pole of $\widetilde{F}(\kappa)=F\left(\kappa^{2}+v_{m}\right)$ corresponding to any of the countably many possible continuations described above.

2. Meromorphic continuations. Let $G^{-}(x, y ; \lambda)$ denote Green's function for the operator $A-\lambda$, where $x, y \in \Omega$ and $\lambda \in \Gamma_{0}$.

THEOREM 1. (a) $G^{-}(x, y ; \lambda)$ has a meromorphic continuation from $\Gamma_{0}$ onto all of $R_{\infty}$. (b) Suppose $\mathscr{G} \subset\left(v_{m}, v_{m+1}\right)-\Lambda$. Then $\mathscr{S}_{m}(\lambda)$ and each $w_{n}^{-}(x ; \lambda), n=1, \ldots, m$, has a meromorphic continuation from $\mathscr{G}$ onto each sheet, $\Gamma_{n_{1}, \ldots, n_{k}}$, of $R_{\infty}$ across $\left(v_{m}, v_{m+1}\right)$, provided $0 \leqq n_{1}, \ldots, n_{k} \leqq m$.

We shall outline the proof of Theorem 1 as follows. Set $\gamma=\dot{\Omega}-\dot{\Omega} \cap \dot{S}$, $\bar{\gamma}=$ closure of $\gamma$ and $B=C(\bar{\gamma})$. Thus $\eta(x) \in B$ if $\eta(x)$ is a continuous function defined on $\bar{\gamma}$. We set $\|\eta\|_{B}=\max _{x \in \bar{\gamma}}|\eta(x)|$. Note that $\bar{\gamma}$ is compact by our definition of $\Omega$. We define the integral operator $T_{\lambda}$ by

$$
T_{\lambda} \eta(x)=2 \int_{\bar{\gamma}} \eta(y) \frac{\partial}{\partial v_{y}} G_{0}^{-}(x, y ; \lambda) d S_{y}{ }^{4}
$$

for each $\eta(x) \in B, x \in \bar{\gamma}$ and $\lambda \in R_{\infty}$.

LeMMA 1. $T_{\lambda}$ is a compact, analytic B-valued function of $\lambda$ and $\mathscr{T}_{\lambda} \equiv_{\mathrm{df}}$ $\left(T_{\lambda}-I\right)^{-1}$ is a mermorphic B-valued function of $\lambda$ on $R_{\infty}$.

Lemma 1 is the key result needed in the proof of Theorem 1 and follows employing the methods of potential theory as well as a result of Steinberg, [7, Theorem 1]. We denote the poles of $\mathscr{T}_{\lambda}$ on $R_{\infty}$ by $\mathscr{D}$. Set

$$
\hat{T}_{\lambda} \eta(x)=2 \int_{\bar{\gamma}} \eta(y) \frac{\partial G_{0}^{-}(x, y ; \lambda)}{\partial v y} d S y
$$

for each $\eta(x) \in B, \lambda \in R_{\infty}$ and $x \in \Omega$.

\footnotetext{
${ }^{4} G_{0}^{-}(x, y ; \lambda)$ is defined in $\Omega-S$ by (1.4) with each $\eta_{n}(\tilde{x})$ continued across $i$ as an odd function.
} 
Lemma 2. For each $x, y \in \Omega(x \neq y)$ and $\lambda \in R_{\infty}-\mathscr{D}$, we have

$$
G^{-}(x, y ; \lambda)=G_{0}^{-}(x, y ; \lambda)+\hat{T}_{\lambda}\left(\eta_{\lambda}(\cdot, y)\right)(x),
$$

where $\eta_{\lambda}\left(\chi^{\prime}, y\right)=-\mathscr{T}_{\lambda}\left(G_{0}^{-}(\cdot, y ; \lambda)\right)\left(x^{\prime}\right), x^{\prime} \in \gamma$.

Lemma 2 follows from the properties of $G_{0}^{-}(x, y ; \lambda)$ as well as results from potential theory. An analogue of Lemma 2 follows for each $w_{n}^{-}(x ; \lambda)$ in the same way. This combined with Lemma 1 and (1.1)-(1.3) implies Theorem 1. The poles of each of the functions $w_{n}^{-}(x ; \lambda), \mathscr{S}_{m}(\lambda)$ and $G^{-}(x, y ; \lambda)$ belong to $\mathscr{D}$. We remark that we can also derive the meromorphic continuation of $\mathscr{S}_{m}(\lambda)$ in an easier way without employing the operator $T_{\lambda}$. The detailed proofs of all of the results of this note will appear elsewhere.

3. Resonant states. We now characterize the poles of $\mathscr{S}_{m}(\lambda)$ in terms of "resonant states".

Definition. Suppose that $m$ is a fixed positive integer, $\lambda_{0} \in \Gamma_{n_{1}, \ldots, n_{k}}$, $1 \leqq n_{1}, \ldots, n_{k} \leqq m$, and there exists a nontrivial solution, $w\left(x ; \bar{\lambda}_{0}\right)$, of

$$
\left(\Delta+\bar{\lambda}_{0}\right) w\left(x ; \bar{\lambda}_{0}\right)=0 \quad \text { in } \Omega, \quad w\left(x ; \bar{\lambda}_{0}\right)=0 \quad \text { on } \dot{\Omega},
$$

where $\bar{\lambda}_{0}$ denotes that value of $\bar{\lambda}_{0}$ in $\Gamma_{n_{1}, \ldots, n_{k-1}}$. Suppose also that there exist constants $c_{n}, n=1,2, \ldots$, with some $c_{j} \neq 0,1 \leqq j \leqq m, j \neq$ $n_{1}, \ldots, n_{k-1}$ such that

$$
\begin{aligned}
w\left(x ; \bar{\lambda}_{0}\right)= & \sum_{n=1}^{m} c_{n} \exp \left\{i\left(\bar{\lambda}_{0}-v_{n}\right)^{1 / 2} x_{N}\right\} \eta_{n}(\tilde{x}) \\
& +\sum_{n=m+1}^{\infty} c_{n} \exp \left\{-i\left(\bar{\lambda}_{0}-v_{n}\right)^{1 / 2} x_{N}\right\} \eta_{n}(\tilde{x})
\end{aligned}
$$

for $x_{N} \geqq \stackrel{\circ}{x}_{N}$. Then we shall say that $\lambda_{0}$ is a $\Gamma_{n_{1}, \ldots, n_{k}}$ resonant state for $A_{\mathscr{G}}(\mathscr{G}$ as in Theorem 1$)$.

Note that $w\left(x ; \bar{\lambda}_{0}\right)$ is exponentially blowing up for $x_{N}$ large.

THEOREM 2. Let $\Gamma_{n_{1}, \ldots, n_{k}}$ denote an arbitrary sheet of $R_{\infty}$ such that $1 \leqq n_{1}, \ldots, n_{k} \leqq m$. Suppose that $\mathscr{G}$ is the interval of Theorem 1(b), $\lambda_{0} \in \Gamma_{n_{1}, \ldots, n_{k}}$ and $\bar{\lambda}_{0}$ is chosen so that $\bar{\lambda}_{0} \in \Gamma_{n_{1}, \ldots, n_{k-1}}$. Then $\lambda_{0}$ is a pole of $\mathscr{S}_{m}(\lambda)$ if and only if $\lambda_{0}$ is a $\Gamma_{n_{1}, \ldots, n_{k}}$ resonant state for $A_{\mathscr{G}}$.

Theorem 2 is proved by obtaining explicit formulas relating the resonant states and the $\mathscr{S}_{m}(\lambda)$, employing the techniques of $[8, \S 3]$. In a future publication, we shall give concrete examples of resonant states. These will be obtained from the theory of waveguides.

4. Perturbations due to a potential. Now suppose that $A$ is the operator given by $-\Delta+q(x)$. associated with the zero Dirichlet boundary 
condition in $S$. It will be proved elsewhere, again employing the integral equation method, that analogues of Theorems 1 and 2 hold for $A_{0}$ and $A$, provided the real-valued potential $q(x)$ satisfies the condition: (C) $q(x) \in$ $L_{2} \operatorname{loc}(S)$ and $|q(x)| \leqq K e^{-\alpha\left|x_{N}\right|}$ for $x_{N} \geqq \stackrel{\circ}{x}_{N}$ and positive constants $K$ and $\alpha$.

In this case the meromorphic continuations onto the sheet $\Gamma_{n_{1}, \ldots, n_{k}}$ are only valid in the intersection, $\bigcap_{j=1}^{k} M_{n_{j}}$, of the strips $M_{n_{j}} \equiv_{\mathrm{df}}$ $\left\{\lambda|| \operatorname{Im}\left(\lambda-v_{n_{j}}\right)^{1 / 2} \mid<\alpha / 2\right\}$. Furthermore, equation (3.2) is replaced by an asymptotic relation of the same form and similarly for the radiation condition (1.1). In the special case in which $q(x)=q\left(x_{N}\right)$, the study of resonant states may be readily replaced by the corresponding problem for the one-dimensional Schroedinger operator on the interval $[0, \infty)$.

\section{REFERENCES}

1. C. Goldstein, Eigenfunction expansions associated with the Laplacian for certain domains with infinite boundaries. I, Trans. Amer. Math. Soc. 135 (1969), 1-31. MR 38 \#2459.

2. - - Eigenfunction expansions associated with the Laplacian for certain domains with infinite boundaries. II. Applications to scattering theory, Trans. Amer. Math. Soc. 135 (1969), 33-50. MR 38 \#2460.

3. - Analytic perturbations of the operator $-\Delta$, J. Math. Anal. Appl. 25 (1969), 128-148. MR 40 \# 578.

4. P. D. Lax and R. S. Phillips, Scattering theory, Pure and Appl. Math., vol. 26, Academic Press, New York, 1967. MR 36 \# 530.

5. N. Shenk and D. Thoe, Eigenfunction expansions and scattering theory for perturbations of $-\Delta$, Rocky Mountain J. Math. 1 (1971), no. 1, 89-125. MR 44 \#4396.

6. A. A. Samarskii and A. N. Tihonov, On excitation of radio wave guides, $\mathrm{Z}$. Tekh. Fiz. 17 (1947), 1283

7. S. Steinberg, Meromorphic families of compact operators, Arch. Rational Mech. Anal. 31 (1968/69), 372-379. MR 38 \#1562.

8. C. Goldstein, Scattering theory for elliptic differential operators in unbounded domains, Math. Research Center Tech. Summary Report \# 1218, University of Wisconsin, Madison, Wis., 1972.

Applied Mathematics Department, Brookhaven National laboratory, Upton, NeW YORK 11973 